\title{
Exploration of gamma radiation shielding features for titanate bismuth borotellurite glasses using relevant software program and Monte Carlo simulation code
}

\begin{abstract}
In this work, gamma radiation shielding parameters for six titanate bismuth borotellurite glasses were investigated. The mass attenuation coefficients $(\mu / \rho)$ have been calculated using XCOM software and MCNP5 code within the photon energy range $0.015-10 \mathrm{MeV}$. The $(\mu / \rho)$ values were then used to calculate the effective atomic number (Zeff), electron density (Ne), mean free path (MFP) and half-value layer (HVL) values. By using the Geometric progression (G-P) method, the exposure buildup factor (EBF) values at $0.015 \mathrm{MeV}-15 \mathrm{MeV}$ photon energy range, with penetration depths up to $40 \mathrm{mfp}$ at intervals $1,5,10,20,30$, and 40 mfp were evaluated. The $30 \mathrm{TeO} 2-30 \mathrm{~B} 2 \mathrm{O} 3-30 \mathrm{Bi} 2 \mathrm{O} 3-10 \mathrm{TiO} 2(\mathrm{~mol} \%)$ glass possesses better gamma ray shielding effectiveness due to a higher value of $(\mu / \rho)$, Zeff and lower values of HVL and MFP. The studied glasses exhibit excellent gamma ray shielding features compared to different types of concretes.
\end{abstract}

Keyword: Radiation shielding; XCOM; MCNP5 code; Mass attenuation coefficient; Mean free path; Half-value layer 